\title{
Optical and magnetic properties of $\mathrm{Zn}_{0.98} \mathrm{Mn}_{0.02} \mathrm{O}$ nanoparticles
}

\author{
Talaat M. Hammad $\cdot$ S. Griesing $\cdot$ M. Wotocek $\cdot$ \\ S. Kuhn · R. Hempelmann • U. Hartmann • \\ Jamil K. Salem
}

Received: 21 February 2012/ Accepted: 7 March 2012/Published online: 25 March 2012

(C) The Author(s) 2012. This article is published with open access at Springerlink.com

\begin{abstract}
Pure and Mn-doped colloidal $\mathrm{ZnO}$ particles were prepared in a solvo-thermal via sol-gel process by base-catalyzed hydrolysis of zinc acetate. We have studied the structural, magnetic and optical properties of the samples using X-ray diffraction (XRD), transmission electron microscopy, energy dispersive X-ray analysis, superconducting quantum interferometer device and UV-Vis spectroscopy. The XRD spectra show that all the samples are hexagonal wurtzite structures. The calculated average particle size of the samples was approximately 7-3 nm, indicating that the particle size decreased by doping with manganese. Magnetic investigations showed that at room temperature the $\mathrm{Mn}$-doped $\mathrm{ZnO}$ possessed ferromagnetism with the saturation magnetic moment of $0.194 \mathrm{emu} / \mathrm{g}$. The room temperature PL measurements illustrate UV-emission centered at $351 \mathrm{~nm}(3.53 \mathrm{eV})$, which is ascribed to the near-band-edge emissions of $\mathrm{ZnO}$, violet emission at $512 \mathrm{~nm}(2.42 \mathrm{eV})$. The UV-Vis spectra showed a blueshift from 3.42 to $3.78 \mathrm{eV}$ when the $\mathrm{ZnO}$ doped with manganese.
\end{abstract}

\section{T. M. Hammad ( $\square)$}

Department of Physics, Al-Azhar University,

P. O. Box 1244, Gaza, Palestine

e-mail: talaat@yahoo.com

S. Griesing · U. Hartmann

Department of Experimental Physics, Saarland University, 66123 Saarbrucken, Germany

M. Wotocek · S. Kuhn $\cdot$ R. Hempelmann

Department of Physical Chemistry,

Saarland University, 66123 Saarbrucken, Germany

J. K. Salem

Department of Chemistry, Al-Azhar University,

P. O. Box 1244, Gaza, Palestine
Keywords Structural $\cdot$ Optical $\cdot$ Magnetic . Mn-doped $\mathrm{ZnO} \cdot$ Nanoparticles

\section{Introduction}

Oxide-based dilute magnetic semiconductors (DMS) have attracted considerable attention because of the possibility of incorporating ferromagnetism in a semiconducting oxide. The metal oxide, $\mathrm{ZnO}$, a wideband-gap semiconductor with a large exciton binding energy of $60 \mathrm{meV}$ at room temperature, is an exceptionally important material for the envisaged applications in many technologies, such as solar energy conversion and optoelectronic devices (Hammad et al. 2010). Of late, much experimental and theoretical research is focused on DMS based on transition metals (TM), such as $\mathrm{Mn}, \mathrm{Ni}$ and Co-doped $\mathrm{ZnO}$, because of their expected potential applications in future spintronics (Bauer et al. 2001). Interestingly in these systems, the origin of ferromagnetism remains an issue of debate, and there is a great deal of controversy over the origin of ferromagnetism. Room temperature ferromagnetism (Sharma et al. 2003; Cong et al. 2005; Schwartz et al. 2004; Radovanovic and Gamelin 2003; Barick and Bahadur 2007; Wang et al. 2007) is observed in several TM-doped $\mathrm{ZnO}$ materials. There are also reports of ferromagnetism only at low temperature (Jung et al. 2002; Wakano et al. 2001) spin glass (Fukamura et al. 2001), and paramagnetic (Kolesnik et al. 2004; Rao and Deepak 2005; Yin et al. 2005; Clavel et al. 2007) behavior in these systems. While the controversy is obvious, the properties seem to be a strong function of the conditions of preparation. Furthermore, most of the previous investigations have focused on thin films and bulk, and there are much less reports on 
nanoparticles of transition metal-doped $\mathrm{ZnO}$. Studies have been carried out to fine-tune the properties of $\mathrm{ZnO}$ to adopt it for different applications; for e.g., the band gap of $\mathrm{ZnO}$ is modified to use as UV detectors and emitters (Tang et al. 1998). Doping Mn into the bulk ZnO matrix offers an interesting way to alter various properties (Fukamura et al. 1999), for example, the band gap of the host material can be tuned from 3.3 to $3.7 \mathrm{eV}$. In addition, it also alters the emission properties by providing an efficient channel for the recombination of the electron and the hole via the dopant Mn d levels. The optical and electronic properties of semiconductors can be further tuned by varying the size of the particles in the range below $10 \mathrm{~nm}$. In addition, it is recently reported (Sharma et al. 2009) that Mn-doped $\mathrm{ZnO}$ thin films as well as in bulk exhibit ferromagnetism at room temperature. Studies on various Mn-doped semiconductor nanocrystals (Levy et al. 1996) have revealed that the properties of these samples, like the band gap, are influenced by the quantum confinement of electronic states; accordingly, these properties of the doped nanocrystals are considerably different compared to those of the doped bulk system (Sapra et al. 2002). These interesting changes in confined DMSs have enlarged the scope of research activity in this field, prompting us to study the confinement effects in Mn-doped $\mathrm{ZnO}$ nanocrystals.

The aim of this work is to evaluate the effect of $\mathrm{Mn}$ doping on the microstructure, magnetic and the optical properties of the powder grown by simple solvo-thermal method. In this letter, we report the changes in the optical properties of $\mathrm{ZnO}$ nanocrystals brought about by successful doping with $\mathrm{Mn}$ ions.

\section{Experiment procedure}

The dispersed $\mathrm{ZnO}$ nanoparticles were prepared according to the route described in Spanhel and Anderson (1991). In our case, the synthesis was carried out under ambienttemperature conditions. Typically $9.8 \mathrm{mmol} \mathrm{Zn}(\mathrm{OAC})_{2} \cdot 2 \mathrm{H}_{2} \mathrm{O}$ and $0.2 \mathrm{mmol} \mathrm{Mn}(\mathrm{OAC})_{2} \cdot 4 \mathrm{H}_{2} \mathrm{O}$ were dissolved at $70{ }^{\circ} \mathrm{C}$ in $200 \mathrm{~mL}$ ethanol under stirring, subsequently cooled to $50{ }^{\circ} \mathrm{C}$. In a second flask, $5.0 \mathrm{mmol} \mathrm{LiOH} \cdot \mathrm{H}_{2} \mathrm{O}$ were dissolved under ultra sound in $200 \mathrm{~mL}$ ethanol (Dong et al. 2005). $\mathrm{LiOH} \cdot \mathrm{H}_{2} \mathrm{O}$ dissolved in ethanol was added dropwise under stirring during $1 \mathrm{~h}$ to the $\mathrm{Zn} \mathrm{Mn}$ acetate solution. The product was stirred for another hour at $50{ }^{\circ} \mathrm{C}$ and cooled down to room temperature. After that the solution was absolutely clear. $800 \mathrm{~mL} n$-hexane were added to precipitate the doped $\mathrm{ZnO}$. Then, the precipitate of doped $\mathrm{ZnO}$ was separated by centrifuging at 4,500 rpm for $1 \mathrm{~h}$. The obtained precipitate was washed twice with $100 \mathrm{~mL}$ ethanol, $300 \mathrm{~mL}$ $n$-hexane and centrifuged at $4,500 \mathrm{rpm}$ for $3 \mathrm{~h}$, and then drying at $60{ }^{\circ} \mathrm{C}$ for $3 \mathrm{~h}$.
The UV absorption spectra were taken with a Hewlett Packard8453 spectrometer. The fluorescence measurements were done with a Perkin-Elmer LS 50B luminescence spectrometer. The transmission electron microscopy (TEM) analysis was done with a Tecnai F300 transmission electron microscope, images taken after suspending in $95 \%$ ethanol. Crystal structure identification and crystal size analysis were carried out by X-ray diffraction (XRD) XDS 2000, Scintac Inc., USA with $\mathrm{CuK} \alpha$ radiation source, and scan rate of $2 \% \mathrm{~min}$. The morphology of the film is characterized by atomic force microscopy (AFM). Magnetic characterization was carried out using a superconducting quantum interferometer device (SQUID), Quantum Design MPMS XL-7 magnetometer in RSO mode.

\section{Results and discussion}

Powder XRD patterns of undoped $\mathrm{ZnO}$ and $\mathrm{Mn}$-doped $\mathrm{ZnO}$ nanoparticles are shown in Fig. 1. The diffraction peaks in Fig. 1 could readily indexed the hexagonal phase of $\mathrm{ZnO}$, the lattice parameters of which were all consistent with the reported values (JPCDS-1451). It is evident from the XRD data that there are no extra peaks due to manganese metal, other oxides or any zinc manganese phase, indicating that the as-synthesized samples are single phase. The Mn ion was understood to have substituted the $\mathrm{Zn}$ site without changing the wurtzite structure. The Debye-Scherrer equation gives the relation between the mean particle size and the full-width of a peak at half-maximum (FWHM) in a polycrystalline sample; it can be expressed as

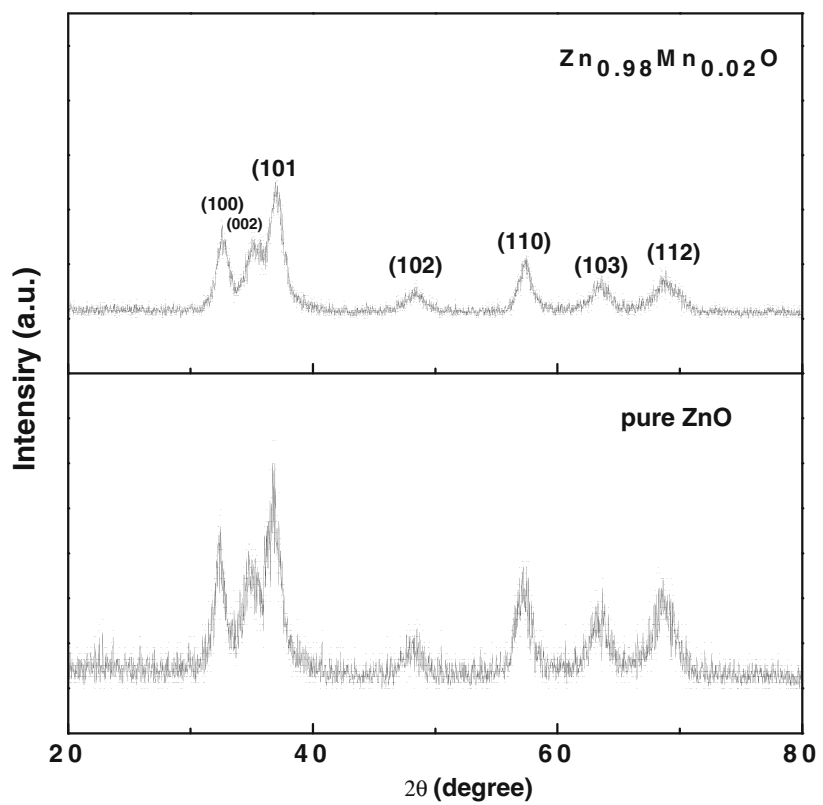

Fig. 1 X-ray diffraction patterns of pure and $\mathrm{Zn}_{0.98} \mathrm{Mn}_{0.02} \mathrm{O}$ nanoparticles 
$d=\frac{0.89 \lambda}{(\beta \cos \theta)}$

where $\mathrm{d}, \lambda, \theta$ and $\beta$ denote the mean grain size, the X-ray wavelength (1.5406 $\AA$ ), the Bragg diffraction angle, and the FWHM, respectively. The calculated average particle size of the samples was approximately 3 and $7 \mathrm{~nm}$, with smaller particle size values for the samples doped with manganese. This may be due to the ionic radius of $\mathrm{Mn}^{2+}$ $(0.83 \AA)$ that is larger than the ionic radius of $\mathrm{Zn}^{2+}$ (0.74 ̊).

The morphology of Mn-doped $\mathrm{ZnO}$ nanoparticles was investigated by high-resolution TEM. Figure 2 shows the TEM images of pure and $\mathrm{Zn}_{0.98} \mathrm{Mn}_{0.02} \mathrm{O}$ nanoparticles.
TEM images of samples were uniform with well-distributed spherical or elliptical particles with a particle size between 3 and $7 \mathrm{~nm}$. This result strongly suggested that $\mathrm{Mn}$ atoms were incorporated uniformly into the entire material and no segregated metal impurity phase appeared. This is consistent with estimated particle size from the XRD peak broadening. The result of HRTEM characterizations are shown in Fig. 3a and b. From HRTEM images, it is clearly observed that the average particle size of $\mathrm{Zn}_{0.98} \mathrm{Mn}_{0.02} \mathrm{O}$ nanoparticles was about $3 \mathrm{~nm}$, which is consistent with the calculation results from XRD. It is also clear from HRTEM results that $\mathrm{Mn}$ doping in $\mathrm{ZnO}$ reduces the particle size. The average interfringe distance of $\mathrm{Zn}_{0.98} \mathrm{Mn}_{0.02} \mathrm{O}$ nanoparticles was measured to be
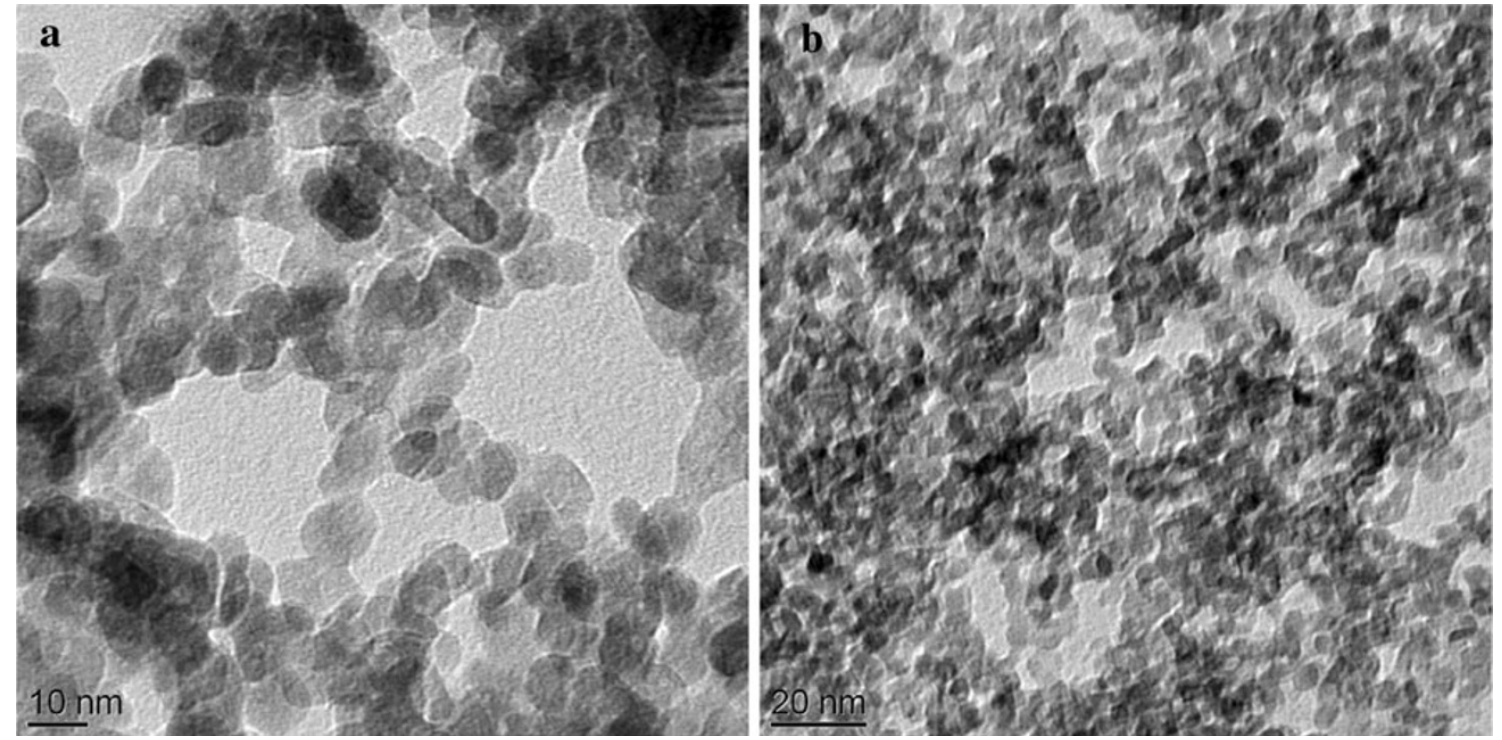

Fig. 2 TEM micrographs of pure and $\mathrm{Mn}$-doped $\mathrm{ZnO}$ : a pure $\mathrm{ZnO}$ and $\mathbf{b} \mathrm{Zn}_{0.98} \mathrm{Mn}_{0.02} \mathrm{O}$ nanoparticles
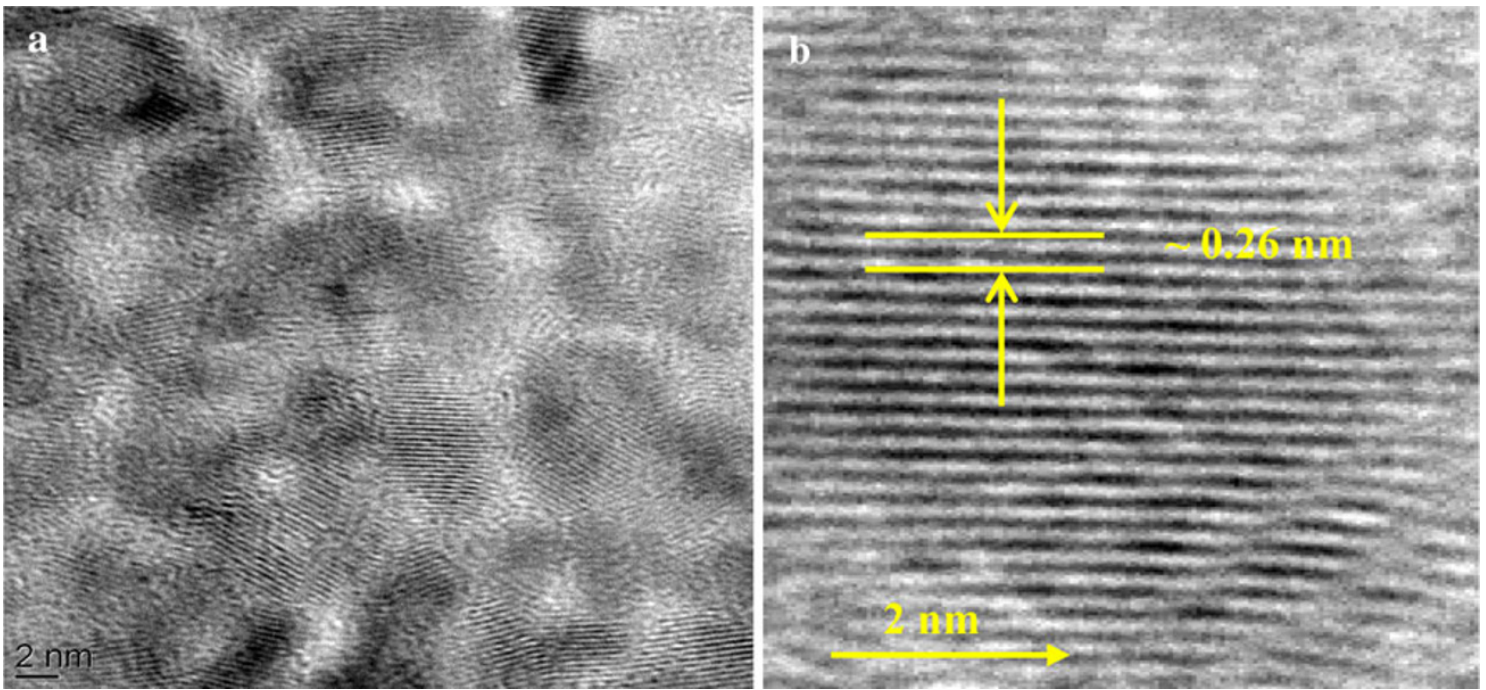

Fig. 3 a, b HRTEM images of $\mathrm{Zn}_{0.98} \mathrm{Mn}_{0.02} \mathrm{O}$ nanoparticles

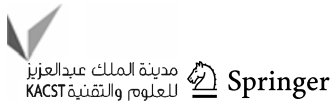


$\sim 0.26 \mathrm{~nm}$. According to the results of XRD pattern and HRTEM images, we believed that the Mn ions were well incorporated into the crystal lattice of $\mathrm{ZnO}$. The EDX analysis of $\mathrm{Mn}$-doped $\mathrm{ZnO}$ and $\mathrm{Zn}_{0.98} \mathrm{Mn}_{0.02} \mathrm{O}$ nanoparticles is shown in Fig. 4. The EDX spectrum confirms the presence of manganese in the $\mathrm{ZnO}$ particles and observed atomic percentage (\%) is close to the nominal value of $\mathrm{Mn}$ in $\mathrm{ZnO}$. Table 1 lists the atomic percentages of the elements present in the Mn-doped $\mathrm{ZnO}$ sample.

The effect of Mn doping on the band gap of $\mathrm{ZnO}$ and substitution of $\mathrm{Mn}^{2+}$ ions in tetrahedral sites of wurtzite structure $\mathrm{ZnO}$ was further confirmed using UV-Vis optical spectroscopy measured at room temperature. Figure 5 shows the UV-absorption spectra of pure $\mathrm{ZnO}$ and $\mathrm{Mn}$-doped $\mathrm{ZnO}$ nanoparticles. The absorption band edge of pure $\mathrm{ZnO}$ is observed at $363 \mathrm{~nm}$ and corresponds to a band gap of $3.42 \mathrm{eV}$, indicating a blue shift of about $0.36 \mathrm{eV}$ as compared to the band gap of Mn-doped $\mathrm{ZnO}$ of $3.78 \mathrm{eV}(328 \mathrm{~nm}$ ). Many other researchers (Rusu et al. 2010; Srinivasan and Kumar 2008; Fukamura et al. 2001; Chikoidze et al. 2007; Wang et al. 2006) reported the blue shift of the fundamental absorption edge with the increase of the $\mathrm{Mn}$ content in the doped $\mathrm{ZnO}$ films and the presence of an additional absorption band in front of the absorption edge. This optical behavior is attributed mainly to the sp-d spin exchange interaction between

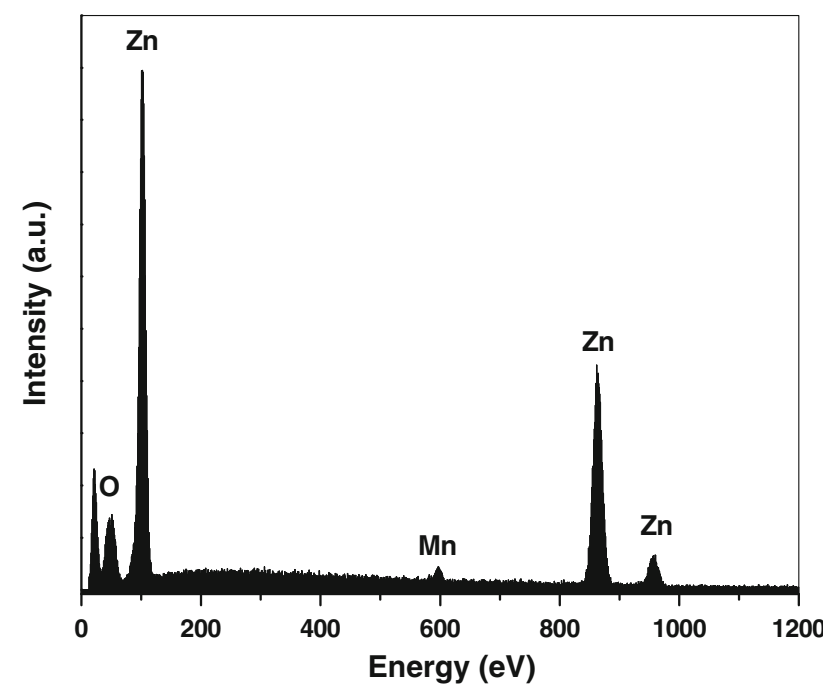

Fig. 4 EDX spectrum of $\mathrm{Zn}_{0.98} \mathrm{Mn}_{0.02} \mathrm{O}$ nanoparticles

Table 1 The atomic percentage calculated for $\mathrm{Zn}_{0.98} \mathrm{Mn}_{0.02} \mathrm{O}$ nanoparticles

\begin{tabular}{lc}
\hline Element & \multicolumn{1}{l}{$\begin{array}{l}\text { Atomic } \\
\text { percentage (\%) }\end{array}$} \\
\hline $\mathrm{Mn}$ & $1.47 \pm 0.33$ \\
$\mathrm{Zn}$ & $48.06 \pm 0.38$ \\
\hline
\end{tabular}

the band electrons and the localized d electrons of $\mathrm{Mn}^{+2}$ ions.

Room temperature photoluminescence (PL) spectra of pure $\mathrm{ZnO}$ and $\mathrm{Mn}$-doped $\mathrm{ZnO}$ samples were measured using photoluminescence spectroscopy. Figure 6 shows the $\mathrm{PL}$ spectra of pure $\mathrm{ZnO}$ and of $\mathrm{Zn}_{0.98} \mathrm{Mn}_{0.02} \mathrm{O}$ at an excitation wavelength of $320 \mathrm{~nm}$. The PL spectra (Fig. 6) consist of two UV emission peaks which centered at $351 \mathrm{~nm}(3.53 \mathrm{eV})$ and $342 \mathrm{~nm}(3.61 \mathrm{eV})$, respectively, and a broad green emission band centered at $512 \mathrm{~nm}(2.42 \mathrm{eV})$. With manganese doped into the $\mathrm{ZnO}$ crystal lattice, the intensity of the peaks for the UV and green emission is reduced. Mn doping is expected to cause an reduction in the intensity of both UV (Hammad et al. 2009) and green emission (Hammad et al. 2009; Salem et al. 2009). It can be found that the optical band gap is increased when $\mathrm{Mn}$

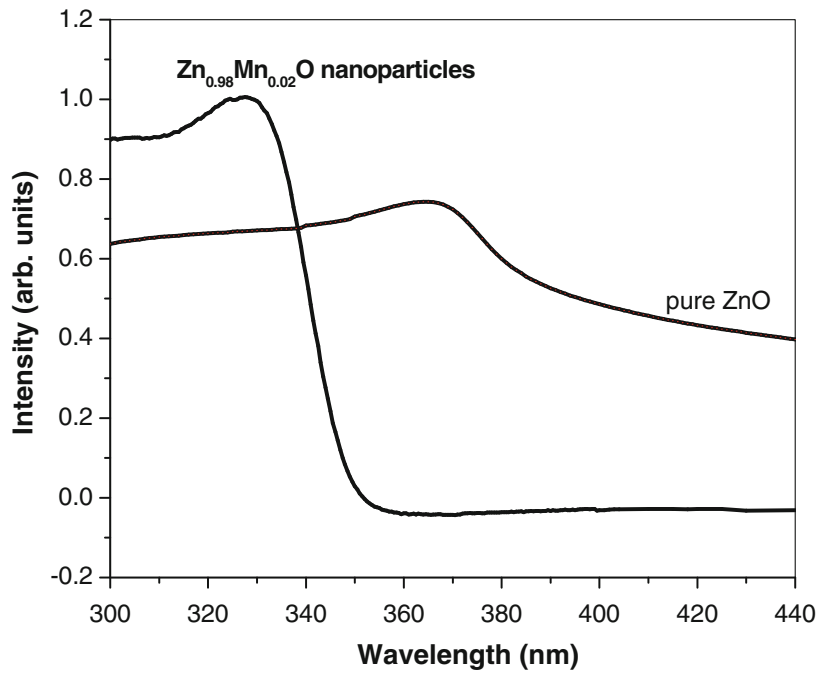

Fig. 5 UV-Vis spectra of pure and $\mathrm{Zn}_{0.98} \mathrm{Mn}_{0.02} \mathrm{O}$ nanoparticles

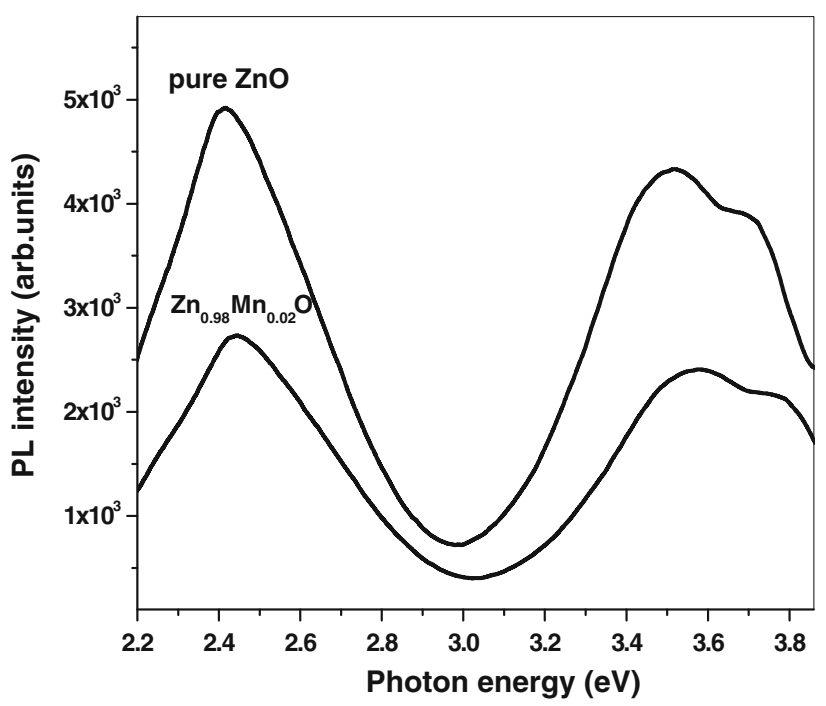

Fig. 6 PL spectra of pure and $\mathrm{Zn}_{0.98} \mathrm{Mn}_{0.02} \mathrm{O}$ nanoparticles 
was doped in $\mathrm{ZnO}$ nanoparticles. Since the band gap of $\mathrm{MnO}$ (bulk value $4.2 \mathrm{eV}$ ) is higher than that $\mathrm{ZnO}$ (bulk value $3.32 \mathrm{eV}$ ), the band gap of $\mathrm{Mn}$-doped $\mathrm{ZnO}$ should be larger than the band gap of $\mathrm{ZnO}$. A similar observation of enhancement of the band gap of $\mathrm{ZnO}$ with $\mathrm{Mn}$ was also made by Fukumura et al. (2001) and Yan et al. (2011). It is commonly considered that UV emission of the wide band gap $\mathrm{ZnO}$ semiconductor attributed to exciton transitions from localized levels is below the conduction band to the valence band (Wang et al. 2006; Huang et al. 2001; Cho et al. 2002; Zu et al. 1997). The localized level assumed to originate from the free impurity atoms and various defects related to oxygen and zinc. Moreover, the origin of the defect-related visible emission in $\mathrm{ZnO}$ nanomaterials may be ascribed to the oxygen vacancies and the intrinsic defects (Li et al. 2006; Sun et al. 2006). The green emission observed in the visible region may be attributed to the impurity levels correspond to the singly ionized oxygen vacancy in $\mathrm{ZnO}$ (Monticone et al. 1998a, b).

To study how the concentration of $\mathrm{Mn}$ atom changes the magnetic behavior of our Mn-doped $\mathrm{ZnO}$ nanoparticles, a magnetization $(\mathrm{M})$ versus applied field $(\mathrm{H})$ study was performed at room temperature using superconducting quantum interferometer. Figure 7 shows the magnetic hysteresis $(\mathrm{M}-\mathrm{H})$ curves of pure $\mathrm{ZnO}$ and $\mathrm{Zn}_{0.98} \mathrm{Mn}_{0.02} \mathrm{O}$ nanoparticles measured at room temperature. The hysteresis loop reveals that the saturation magnetization Ms and the coercivity $\mathrm{Hc}$ of pure and doped $\mathrm{ZnO}$ nanoparticles are about $0.014 \mathrm{emu} / \mathrm{g}, 4.3 \mathrm{Oe}, 00.194 \mathrm{emu} / \mathrm{g}$ and $272 \mathrm{Oe}$, respectively. Furthermore, the doped $\mathrm{ZnO}$ sample exhibits higher magnetization values than that of the pure $\mathrm{ZnO}$ sample. This magnetization of present doped $\mathrm{ZnO}$ is larger

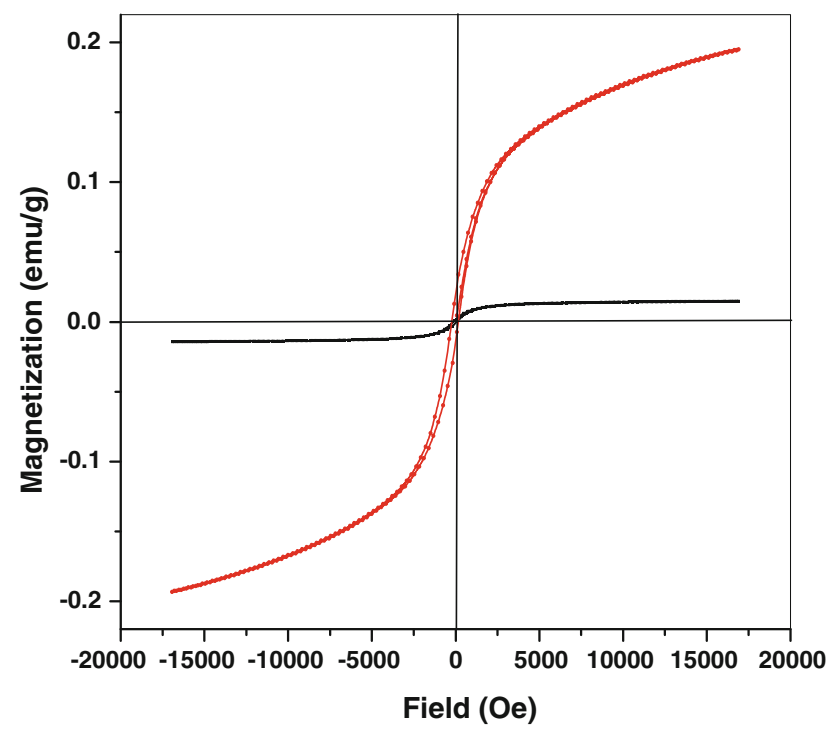

Fig. 7 Magnetization of Mn-doped $\mathrm{ZnO}$ nanoparticle as a function of field measured at room temperature than values were reported by Jayakumar et al. 2007 and Ma and Lou1 (2011). Jayakumar et al. 2007 have observed that the value of magnetization of $2.5 \%$ Mn-doped $\mathrm{ZnO}$ nanoparticles at room temperature is $0.034 \mathrm{emu} / \mathrm{g}$.

However, Ma and Lou1 (2011) estimated the magnetization of $10 \%$ Mn-doped $\mathrm{ZnO}$ nanoparticles to be $0.015 \mathrm{emu} / \mathrm{g}$.

Ferromagnetism in dilute magnetic semiconductors is considered to originate from the exchange interaction between free delocalized carriers (holes or electrons from the valence band) and the localized d spins on the TM ions (Dietl et al. 2000). Therefore, the presence of free carriers and localized $\mathrm{d}$ spins is a prerequisite for the appearance of ferromagnetism. These defects like $\mathrm{Zn}$ interstitials and $\mathrm{O}$ vacancies usually induce $n$-type characteristics. The mechanism for intrinsic ferromagnetism in $\mathrm{Zn}_{1-x} \mathrm{Mn}_{x} \mathrm{O}$ thin films still remains unclear, and the source of ferromagnetism remains controversial. However, detailed structural characterization minimizes the possibility that the ferromagnetism is due to any impurity phases in our samples. It has been proposed that uncontrolled formation of lattice defects can generate carriers that mediate ferromagnetic behavior (Venkatesan et al. 2004; Coey et al. 2005). Recently, Sundaresan et al. (2006) observed that metal oxides such as $\mathrm{CeO}_{2}, \mathrm{Al}_{2} \mathrm{O}_{3}, \mathrm{ZnO}, \mathrm{In}_{2} \mathrm{O}_{3}$ and $\mathrm{SnO}_{2}$ in nanoparticulate form exhibit room temperature ferromagnetism presumably due to the exchange interaction between localized spin moments resulting from the oxygen vacancies at the surface of nanoparticles. The native point defects such as oxygen vacancies are very common in $\mathrm{ZnO}$ nanoparticles giving rise to ferromagnetic behavior. Interestingly, as compared to single nanoparticles, these selfaggregated $\mathrm{ZnO}$ superstructure exhibits an higher magnetization, which may be due to the formation of higher amount of defects as a result of oriented attachment among nanocrystals. In comparison to pure $\mathrm{ZnO}$ superstructures, $\mathrm{Mn}$ and $\mathrm{Ni}$ doping may increase the concentration of oxygen vacancy because of their slightly larger ionic radii than $\mathrm{Zn}$ ions.

\section{Conclusion}

Manganese-doped $\mathrm{ZnO}$ nanoparticles have been successfully prepared in a sol-gel process by base-catalyzed hydrolysis of zinc acetate. Microstructure analysis confirms the single phase hexagonal wurtzite structure for Mn-doped $\mathrm{ZnO}$ and spherical nanoparticles were observed through TEM images. The average particle size calculated from XRD patterns of the samples was approximately $7-3 \mathrm{~nm}$, indicating that the particle size decreased by doping with manganese. Magnetic investigations showed that the Mndoped $\mathrm{ZnO}$ nanoparticles possessed room temperature 
ferromagnetism with the saturation magnetic moment of $0.1 \mathrm{emu} / \mathrm{g}$. The room temperature PL spectrum of the $\mathrm{Zn}_{0.98} \mathrm{Mn}_{0.02} \mathrm{O}$ nanoparticles consisted of a strong $\mathrm{UV}$ NBE emission band at $351 \mathrm{~nm}$ and a broad green emission at around $530 \mathrm{~nm}$. The UV NBE emission of the $\mathrm{Zn}_{0.98} \mathrm{Mn}_{0.02} \mathrm{O}$ nanoparticles showed a blue shift owing to Mn substitution.

Acknowledgments The authors would like to gratefully appreciate the financial support from the DAAD and Saarland University, Germany.

Open Access This article is distributed under the terms of the Creative Commons Attribution License which permits any use, distribution, and reproduction in any medium, provided the original author(s) and the source are credited.

\section{References}

Barick KC, Bahadur DJ (2007) Synthesis, self-assembly, and properties of $\mathrm{Mn}$ doped $\mathrm{ZnO}$ nanoparticles. J Nanosci Nanotechnol 7:1935-1940

Bauer C, Boschloo G, Mukhtar E, Hagfeldt A (2001) Electron injection and recombination in $\mathrm{Ru}(\mathrm{dcbpy})_{2}(\mathrm{NCS})_{2}$ sensitized nanostructured ZnO. A J Phys Chem B 105:5585-5588

Chikoidze E, Dumont Y, Jomard F, Gorochov O (2007) Electrical and optical properties of $\mathrm{ZnO}: \mathrm{Mn}$ thin films grown by MOCVD. Thin Solis Films 515:8519-8523

Cho YM, Choo WK, Kim H, Kim D, Ihm YE (2002) Effects of rapid thermal annealing on the ferromagnetic properties of sputtered $\mathrm{Zn}_{1-x}\left(\mathrm{Co}_{0.5} \mathrm{Fe}_{0.5}\right)_{x} \mathrm{O}$ thin films. Appl Phys Lett 80:3358-3360

Clavel G, Willinger M-G, Zitoun D, Pinna N (2007) Solvent dependent shape and magnetic properties of doped $\mathrm{ZnO}$ nanostructures. Adv Funct Mater 17:3159-3169

Coey JMD, Venkatesan M, Fitzgerald CB (2005) Donor impurity band exchange in dilute ferromagnetic oxides. Nat Mater 4:173179

Cong CJ, Liao L, Li JC, Fan LX, Zhang KL (2005) Synthesis, structure and ferromagnetic properties of Mn-doped $\mathrm{ZnO}$ nanoparticles. Nanotechnol 16:981-984

Dietl T, Ohno H, Matsukura F, Cibert J, Ferrand D (2000) Zener model description of ferromagnetism in zinc-blende magnetic semiconductors. Science 287:1019-1022

Dong L, Liu YC, Tong YH, Xiao ZY, Zhang JY, Lu YM, Shen DZ, Fan XW (2005) Preparation of $\mathrm{ZnO}$ colloids by aggregation of the nanocrystal subunits. J Colloid Interface Sci 15:380-384

Fukamura T, Jin Z, Ohtomo A, Koinuma H, Kawasaki M (1999) An oxide-diluted magnetic semiconductor: Mn-doped ZnO. Appl Phys Lett 75:3366-3369

Fukamura T, Jin Z, Kawasaki M, Shono T, Hasegawa T, Koshihara S, Koinuma H (2001) Magnetic properties of Mn-doped ZnO. Appl Phys Lett 78:958-961

Hammad T, Salem JK, Harrison RG (2009) Synthesis, characterization, and optical properties of Y-doped ZnOnanoparticles. NANO 4:225-232

Hammad T, Salem JK, Harrison RG (2010) The influence of annealing temperature on the structure, morphologies and optical properties of $\mathrm{ZnO}$ nanoparticles. Superlattices Microstruct 47:335-340

Huang MH, Wu YY, Feick HN, Tran N, Weber E, Yang PD (2001) Room-temperature ultraviolet nanowire nanolasers. Adv Mater $13: 113$
Jayakumar OD, Gopalakrishnan IK, Kadam RM, Vinu A, Asthana A, Tyagi AK (2007) Magnetization and structural studies of Mn doped $\mathrm{ZnO}$ nanoparticles: prepared by reverse micelle method. J Cryst Growth 300:358-363

Jung SW, An S-J, Yi G-C, Jung CU, Lee S-I, Cho S (2002) Ferromagnetic properties of $\mathrm{Zn}_{1-x} \mathrm{Mn}_{x} \mathrm{O}$ epitaxial thin films. Appl Phys Lett 80:4561-4564

Kolesnik S, Dabrowski B, Mais J (2004) Structural and magnetic properties of transition metal substituted $\mathrm{ZnO}$. J Appl Phys 95:2582-2587

Levy L, Hochepied JF, Pileni MP (1996) Control of the size and composition of three dimensionally diluted magnetic semiconductor clusters. J Phys Chem 100:18322-18326

Li C, Fang G, Fu Q, Su F, Li G, Wu XZ, Zhao X (2006) Effect of substrate temperature on the growth and photoluminescence properties of vertically aligned $\mathrm{ZnO}$ nanostructures. J Cryst Growth 292:19-25

Ma X, Lou C (2011) The dilute magnetic and optical properties of Mn-doped ZnO nanowires. J Nanomaterial 2011:464538464543

Monticone S, Tufeu R, Kanaev AV (1998a) Complex nature of the $\mathrm{UV}$ and visible fluorescence of colloidal $\mathrm{ZnO}$ nanoparticles. J Phys Chem B 102:2854-2862

Monticone S, Tufeu R, Kanaev AV (1998b) Prepared in the presence of additives by thermal decomposition. Int J Nanosci 8:465-472

Radovanovic PV, Gamelin DR (2003) High temperature ferromagnetism in nanocrystalline $\mathrm{Ni}^{2+}$-doped $\mathrm{ZnO}$. Phys Rev Lett 92:157202

Rao CNR, Deepak FL (2005) Absence of ferromagnetism in Mn- and Co-doped ZnO. J Mater Chem 15:573-578

Rusu GG, Gorley P, Baban PC, Rambu AP, Rusu M (2010) Preparation and characterization of $\mathrm{Mn}$-doped $\mathrm{ZnO}$ thin films. J Optoelectron Adv Mater 12:895-899

Salem JK, Hammad TM, Harrison RG (2009) ZnO nanoparticles prepared in the presence of additives by thermal decomposition. Int J Nanosci 8:465-472

Sapra S, Sarma DD, Sanvito S, Hill N (2002) Influence of quantum confinement on the electronic and magnetic properties of $(\mathrm{Ga}$, Mn)As diluted magnetic semiconductor. A Nano Lett 2:605-608

Schwartz DA, Kittilstved KR, Gamelin DR (2004) Above roomtemperature ferromagnetic $\mathrm{Ni}^{2+}$-doped $\mathrm{ZnO}$ thin films prepared from colloidal diluted magnetic semiconductor quantum dots. Appl Phys Lett 85:1395-1398

Sharma P, Gupta A, Rao KV, Owens FJ, Sharma R, Ahuja R, Guillen JMO, Johansson B, Gehring GA (2003) Ferromagnetism above room temperature in bulk and transparent thin films of Mn-doped ZnO. Nat Mater 2:673-677

Sharma PK, Dutta RK, Pandey AC, Layek S, Verma HC (2009) Effect of iron doping concentration on magnetic properties of ZnO nanoparticles. J Magn Magn Mater 321:2587-2591

Spanhel L, Anderson MA (1991) Semiconductor clusters in the solgel process: quantized aggregation, gelation, and crystal growth in concentrated zinc oxide colloids. J Am Chem Soc 113(8): 2826-2833

Srinivasan G, Kumar J (2008) Effect of Mn doping on the microstructures and optical properties of sol-gel derived $\mathrm{ZnO}$ thin film. J Crys Growth 310:1841-1846

Sun Y, Ndifor-Angwafor NG, Riley DJ, Ashfold MNR (2006) Synthesis and photoluminescence of ultra-thin $\mathrm{ZnO}$ nanowire. Chem Phys Lett 431:352

Sundaresan A, Bhargavi R, Rangarajan N, Siddesh U, Rao CNR (2006) Ferromagnetism as a universal feature of nanoparticles of the otherwise nonmagnetic oxides. Phys Rev B74:161306(R)

Tang ZK, Wong GKL, Yu P, Kawasaki M, Ohtomo A, Koinuma H, Segawa Y (1998) Blue light-emitting diode based on ZnO. Appl Phys Lett 72:3270 
Venkatesan M, Fitzgerald CB, Lunney JG, Coey JMD (2004) Anisotropic ferromagnetism in substituted zinc oxide. Phys Rev Lett 93:177206-177210

Wakano T, Fujimura N, Abe N, Ashida A, Ito T (2001) Magnetic and magneto-transport properties of $\mathrm{ZnO}: \mathrm{Ni}$ films. Physica $\mathrm{C}$ 10:160-164

Wang YS, Thomas PJ, Brien PO (2006) Optical properties of ZnO nanocrystals doped with $\mathrm{Cd}, \mathrm{Mg}, \mathrm{Mn}$, and $\mathrm{Fe}$ ions. J Phys Chem B 110(43):21412-21415

Wang H, Chen Y, Wang HB, Zhang C, Yang FJ, Duan JX, Yang CP, Xu YM, Zhou MJ, Li Q (2007) High resolution transmission electron microscopy and Raman scattering studies of room temperature ferromagnetic Ni-doped $\mathrm{ZnO}$ nanocrystal. Appl Phys Lett 90(5):052505
Yan X, Hu D, Li H, Li L, Chong X, Wang Y (2011) Nanostructure and optical properties of $\mathrm{M}$ doped $\mathrm{ZnO}(\mathrm{M}=\mathrm{Ni}, \mathrm{Mn})$ thin films prepared by sol-gel process. Physica B Phys Condensed Matter 406:3956

Yin ZG, Chen N, Yang F, Chai SL, Zhong J, Qian HJ, Ibrahim K (2005) Structural, magnetic properties and photoemission study of Ni-doped ZnO. Solid State Commun 135:430-433

Zu P, Tang ZK, Wong GKL, Kawasaki M, Ohtomo A, Koinuma HY, Segawa Y (1997) Ultraviolet spontaneous and stimulated emissions from $\mathrm{ZnO}$ microcrystallite thin films at room temperature. Solid State Commun 103:456-463 